\title{
Glottopol
}

Revue de sociolinguistique en ligne

$34 \mid 2020$

Les « langues de France ", 20 ans après

\section{Le statut constitutionnel des langues régionales en droit comparé. De la reconnaissance à l'indifférence}

Wanda Mastor

\section{(2) OpenEdition}

Journals

Édition électronique

URL : https://journals.openedition.org/glottopol/372

DOI : $10.4000 /$ glottopol.372

ISSN : 1769-7425

Éditeur

Presses universitaires de Rouen et du Havre

Référence électronique

Wanda Mastor, «Le statut constitutionnel des langues régionales en droit comparé. De la reconnaissance à l'indifférence », Glottopol [En ligne], 34 | 2020, mis en ligne le 01 juillet 2020, consulté le 02 octobre 2021. URL : http://journals.openedition.org/glottopol/372 ; DOI : https://doi.org/ 10.4000/glottopol.372

Glottopol 


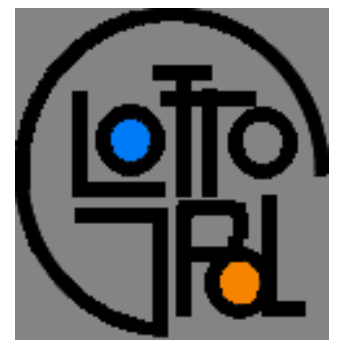

\section{GLOTTOPOL}

Revue de sociolinguistique en ligne $\mathrm{n}^{\circ} 34$ - juillet 2020

Les "langues de France " : 20 ans après

\section{SOMMAIRE}

Hommages à Jean Le Dî

Christian Lagarde : "Langues de France ». Au-delà du symbolique?

Entretien avec Bernard Cerquiglini, par Christian Lagarde.

Entretien avec Paul de Sinety, Délégué général à la langue française et aux langues de France, par Christian Lagarde.

Georg Kremnitz : La problématique initiale de la liste Cerquiglini et ses effets ultérieurs.

Alain Viaut : De "langue régionale » à "langue de France » ou les ombres du territoire.

Wanda Mastor: Le statut constitutionnel des langues régionales en droit comparé. De la reconnaissance à l'indifférence.

Philippe Martel, Marie-Jeanne Verny: Les langues régionales au Parlement, ou l'éternel retour.

Romain Colonna : Les «langues de France »: des langues non-étatiques au pays de l'Étatnation.

Hervé le Bihan : La langue bretonne : une visibilité toute en retenue.

Véronique Bertile : Les langues d'outre-mer : des langues de France? Approche juridique.

Jacques Vernaudon: Les langues polynésiennes et kanak, des "langues de France» en contexte de décolonisation.

Luc Biichlé : Qu'advient-il de l'arabe de France? Mise en perspective sociolinguistique...

Pascal Ottavi : L'épervier, la cage et le passereau.

Marielle Rispail : Le francique lorrain, langue de France? Réflexions et témoignages.

\section{Compte rendu de lecture}

Par Salih Akin : Jean Le Dû \& Yves Le Berre, Métamorphoses. Trente ans de sociolinguistique à Brest (1984-2014), Brest, Centre de Recherche Bretonne, 2019, 302 p.

http://glottopol.univ-rouen.fr 


\title{
LE STATUT CONSTITUTIONNEL DES LANGUES RÉGIONALES EN DROIT COMPARÉ. DE LA RECONNAISSANCE À L'INDIFFÉRENCE
}

\author{
Wanda Mastor \\ Centre de droit comparé, Université Toulouse Capitole
}

La notion de « langues de France » est aussi belle qu'elle revêt, juridiquement, des allures d'oxymore. Pour le dire brutalement, mais conformément à la vérité du droit constitutionnel, le pluriel est plus qu'impossible : il a même été censuré par le Conseil constitutionnel. Plus exactement, ce dernier a censuré la possibilité d'une coexistence juridique de plusieurs langues, la seule langue de la République étant le français. En droit constitutionnel, c'est la seule question des langues régionales qui a fait débat. Mais plutôt que de s'arrêter à ce constat qui ravira les Jacobins attachés au principe de l'unicité de la langue française et décevra les défenseurs du pluralisme linguistique, cette étude propose de tenter de dépasser cette contradiction quasi ontologique. Si elle s'attache à étudier le statut constitutionnel des langues régionales, c'est parce que, d'une part, cette notion, malgré les controverses, a survécu en droit. Et parce que, d'autre part, l'étudier par le prisme du droit comparé permet de mettre en évidence la singularité du système français et de combattre certains arguments dénués de fondements solides. Au premier rang desquels celui en vertu duquel le caractère indivisible de la République entrainerait l'unicité de la langue française. L'étude des constitutions européennes apporte la preuve irréfutable non seulement de la faiblesse, mais aussi de la fausseté de cet argument. En résumé, une étude juridique sur le statut constitutionnel des langues régionales peut être utile à toute étude de plus grande ampleur sur les langues de France; si elle devait porter sur ces dernières ainsi dénommées, elle serait condamnée à demeurer dans les sphères de l'imaginaire.

L'intitulé mérite quelques éclaircissements pour les personnes étrangères au monde parfois hermétique du droit. Le terme de «statut constitutionnel » signifie une essence particulière et emporte des conséquences. Une essence particulière tout d'abord. Seuls des éléments importants peuvent être gravés dans le marbre de la constitution puisque précisément, cette dernière est la plus fondamentale des normes. Le système juridique, selon l'image du théoricien autrichien Hans Kelsen, est une construction pyramidale. Un ensemble de normes hiérarchisées, chaque norme tirant sa validité de sa conformité à la norme supérieure. Concrètement, et au risque de sacrifier quelque peu la précision rigoureuse à l'autel de la pédagogie, cela signifie qu'un acte administratif doit être conforme à la loi qui elle-même doit être conforme à la constitution. D'aucuns seraient tentés de pointer l'aporie d'une telle construction intellectuelle : si un système juridique n'est valide que parce que chaque norme est conforme à une norme supérieure, d'où l'ultime - la constitution - tire-t-elle sa validité ? Kelsen répond en posant l'existence d'une norme hypothétique fondamentale - la Grundnorm-qui coifferait l'ensemble 
du système juridique. Quoi qu'il en soit, cette construction qui fait le cauchemar des étudiants de droit de première année justifie la primauté absolue de la constitution d'un État de droit.

Ces prolégomènes sont importants pour la démonstration qui va suivre, car une personne non juriste peut être en droit de se demander pourquoi la consécration de l'existence d'une langue régionale est plus importante au sein de la constitution plutôt qu'au sein d'une loi. En d'autres termes, une loi ordinaire peut être défaite par une autre loi. En revanche, ce que la constitution fait, seule une révision constitutionnelle peut le défaire. Et il est sensiblement plus difficile d'adopter une loi constitutionnelle (loi qui modifie la constitution, et qui doit être adoptée par les deux tiers de l'Assemblée nationale et du Sénat réunis en Congrès) qu'une simple loi.

Toujours à propos de l'essence particulière que renferme l'hypothèse d'un statut constitutionnel, il faut donc réaliser que la constitution ne doit pas comporter «n'importe quoi » tout comme elle ne doit comporter que l'essentiel. «N'importe quoi » signifie, en termes scientifiques, qu'elle ne doit pas comporter de dispositions qui, matériellement, substantiellement, ne seraient pas de nature constitutionnelle. La constitution doit être, ne devrait être que l'ensemble des règles aménageant l'organisation du pouvoir, le fonctionnement du pouvoir, et les relations gouvernants-gouvernés à travers notamment un catalogue de droits fondamentaux. C'est ce que nous pourrions qualifier de triptyque constitutionnel, toutes les règles relatives à ce triptyque étant matériellement constitutionnelles. Tout État a nécessairement une constitution matérielle, qu'elle soit écrite ou non. Ce qui ne relève pas de ces matières est en revanche d'ordre cosmétique. Les préambules peuvent bien évidemment contenir des symboles, présentés parfois à travers un style quasi lyrique ou épique selon les cultures nationales. Une nation en a également besoin. Mais l'essentiel des dispositions de la constitution doivent revêtir un caractère normatif, c'est-à-dire qu'elles doivent être effectives. Nous ne prendrons ici qu'un seul exemple lié au sujet traité.

Préciser dans la constitution que les langues régionales appartiennent au patrimoine de la France n'a pas de portée normative. Avant que le Conseil constitutionnel ne vienne le confirmer $^{1}$, la rédaction ne laissait déjà pas grand place au doute interprétatif. Le fait que le justiciable ne puisse pas s'en prévaloir n'est pas une faute imputable au seul Conseil constitutionnel, mais aussi - et avant tout - au pouvoir constituant. En rédigeant ainsi le nouvel article 78 de la Constitution, dont on relèvera l'éloignement vis-à-vis de l'article 2 relatif à la langue française, le pouvoir constituant (c'est-à-dire, celui qui révise la Constitution) savait pertinemment qu'il n'emporterait pas de conséquence juridique. Précisons ici qu'en droit constitutionnel, la question du statut constitutionnel désigne non seulement une norme qui serait inscrite dans la constitution, mais aussi dégagée et protégée par la justice constitutionnelle. Dans la plupart des pays européens, et conformément à la philosophie qui légitimât leur création après la seconde guerre mondiale et les errements des parlements, les juges constitutionnels sont les garants des libertés fondamentales.

Le terme de «statut constitutionnel » signifie donc avant tout une essence particulière. Il emporte ensuite des conséquences.

Concrètement, le fait d'inscrire un droit dans la constitution a des effets pratiques. Prenons l'exemple de l'insertion de la Corse dans la Constitution de la $\mathrm{V}^{\mathrm{e}}$ République française, qui demeure très hypothétique au moment où nous écrivons. Les détracteurs de ladite insertion, s'appuyant sur des arguments plus ou moins honnêtes scientifiquement tant ils sont en réalité politiques, répondent que la Corse est déjà une collectivité à statut particulier. Qu'elle bénéficie

\footnotetext{
${ }^{1}$ Décision n ${ }^{\circ}$ 2011-130 QPC du 20 mai 2011, Mme Cécile L. et autres [Langues régionales] : « Considérant qu'aux termes de l'article 75-1 de la Constitution : «Les langues régionales appartiennent au patrimoine de la France »; que cet article n'institue pas un droit ou une liberté que la Constitution garantit ; que sa méconnaissance ne peut donc être invoquée à l'appui d'une question prioritaire de constitutionnalité sur le fondement de l'article 61-1 de la Constitution (...) » (considérant 3).
} 
déjà d'une architecture institutionnelle spécifique et de compétences spécifiques. C'est absolument exact. Mais toutes ces spécificités n'ont, en l'état actuel des choses, qu'un statut législatif. Comme précisé plus haut, une simple loi pourrait défaire tout ce qui a été fait - et parfois, acquis de haute lutte politique. Ce d'autant plus dans un domaine, celui du droit des collectivités territoriales, qui est particulièrement mouvant tant la décentralisation est un sujet idéologique. Un statut constitutionnel de la Corse serait donc une manière de le sanctuariser en lui offrant une protection renforcée.

Venons-en, non plus à la question du niveau de garantie, mais à celle de son objet, les langues régionales. Un tour d'horizon des textes des constitutions européennes et des décisions des cours constitutionnelles permet de mettre en évidence plusieurs éléments significatifs. Le droit comparé nous enseigne en effet que juridiquement, la question des langues s'appréhende à travers deux prismes : premièrement, celui de la forme de l'État. Le lien entre unité de l'État et unilinguisme est aussi évident que celui entre État composé (régional ou fédéral) et multilinguisme. Deuxièmement, celui des droits des locuteurs. Selon cette acception, les langues régionales sont traditionnellement associées, de manière négative, au principe de nondiscrimination; de manière positive, aux droits linguistiques dont les locuteurs seraient les bénéficiaires ${ }^{2}$.

Ne seront abordées dans le cadre de cette étude que les langues régionales du point de vue institutionnel (la forme de l'État) et non substantiel (les droits des locuteurs). La question de la place des langues régionales dans l'espace public est indissociable de celle du degré d'autonomie qu'une constitution accorde à ses territoires. Pour le dire autrement, il est impossible de s'interroger sur les droits des locuteurs si l'État ne permet pas, au préalable, une reconnaissance des langues au pluriel assortie d'effets normatifs. Preuve en est notamment le lien entre la co-officialité et la forme de l'État. La logique du principe de co-officialité, quand il est consacré par les constitutions nationales, est avant tout une logique territoriale. En droit constitutionnel comparé, l'aménagement de la pluralité linguistique diverge selon que les États reconnaissent une pluri-officialité sur l'ensemble du territoire ou au seul niveau local. La première hypothèse est celle naturellement choisie par la Belgique et la Suisse, États fédéraux européens. Selon l'article 4 de la Constitution helvétique, «les langues nationales sont l'allemand, le français, l'italien et le romanche ». De son côté, la Constitution belge énonce que « la Belgique comprend trois communautés: la communauté française, la communauté flamande et la communauté germanophone » et fait également référence à " quatre régions linguistiques : la région de langue française, de région de langue néerlandaise, la région bilingue de Bruxelles-capitale et la région de langue allemande ». En revanche, l'Italie et l'Espagne ne reconnaissent qu'une seule langue officielle sur l'ensemble du territoire en même temps qu'est aménagée, au niveau local, la pluralité linguistique. C'est ce second type d'aménagement qui sera ici étudié, d'une part parce qu'il est plus complexe donc plus passionnant, d'autre part parce que la comparaison de la France avec les États régionaux est plus pertinente scientifiquement que celle avec les États fédéraux. L'État régional est en effet l'une des variantes de l'État unitaire, et il est un parfait exemple de l'absence d'incompatibilité entre le caractère indivisible des États concernés et l'épanouissement en leur sein du multilinguisme. Pour le dire autrement, ils sont la preuve que l'indivisibilité ne doit pas être entendue comme synonyme d'uniformité. Nier la diversité, notamment linguistique, est aussi une manière de nier la richesse de la République ${ }^{3}$.

Il ne peut pas y avoir un statut constitutionnel des droits linguistiques si les langues régionales ne bénéficient pas, a priori, d'un minimum de reconnaissance de même niveau. De

\footnotetext{
${ }^{2}$ Voir le rapport explicatif de de la Charte européenne des langues régionales ou minoritaires, Conseil de l'Europe, Série des Traités européens, ${ }^{\circ} 148$, Strasbourg, 5 novembre 1992, https://rm.coe.int/16800cb620

${ }^{3}$ C'est essentiellement ce lien entre la construction de l'État et les langues régionales qui a été au centre d'un colloque organisé à Toulouse et publié (Gorgorza A. et Mastor W. 2019).
} 
ce point de vue, l'Europe est un formidable laboratoire pour le comparatiste qui éprouve bien des difficultés à opérer une typologie. Même s'il ne s'agit pas de l'étape la plus stimulante intellectuellement, le comparatiste doit commencer par classer des objets, des régimes et des postures avant de les confronter. De manière schématique, plusieurs postures essentielles visà-vis des langues peuvent être observées dans l'Europe contemporaine.

Une première posture de l'État central envers les langues régionales pourrait être qualifiée d'assimilationniste, comme l'a démontré Véronique Bertile dans sa thèse de référence (2008). Dans ce cas, l'État, généralement très centralisateur, utilise des outils destinés à minorer certains groupes linguistiques. Ces outils peuvent aller de la dévalorisation, l'humiliation, à l'interdiction. Au-delà de la problématique de la valorisation, une seule langue est tout simplement « reconnue » sur les plans politique, juridique, social, économique. Une deuxième politique qui peut être observée au niveau européen est celle du statut juridique différencié. Coexistent alors une langue majoritaire et des langues minoritaires, qui bénéficient de mesures protectrices. Dans ce cas, les langues régionales jouissent d'une reconnaissance mais seulement au niveau d'un territoire identifié. Pour le dire autrement, elles ne concurrencent pas la langue majoritaire au niveau national. Il ne s'agit ni plus ni moins que de l'expression d'un droit à la différence. D'un droit à la différenciation, pour reprendre un terme qui a actuellement les faveurs du gouvernement français. Enfin, la troisième politique qui peut être qualifiée de maximaliste est celle du bilinguisme officiel, qui peut s'exprimer dans plusieurs variantes. Ce statut confère aux citoyens, en principe du moins, le choix d'utiliser l'une ou l'autre des langues officielles dans leurs rapports avec l'État. Cette co-officialité peut s'opérer au niveau de tout le territoire (comme en Belgique et en Suisse par exemple) ou seulement sur une partie de celuici (comme en Espagne ou en Italie).

Ces trois politiques linguistiques correspondent en réalité à deux postures générales qui sont fixées dans les constitutions et interprétées par les cours constitutionnelles. Il est en effet possible de distinguer d'une part les constitutions qui sont indifférentes aux langues régionales (II), et d'autre part, celles qui leur offre, à des degrés bien évidemment divers, des formes de reconnaissance (I).

\section{Reconnaitre le plurilinguisme de la société}

C'est, comme toujours, le recours à l'histoire qui permet au comparatiste de mieux saisir son objet. Bien évidemment la question du statut constitutionnel des langues régionales est ontologiquement liée aux évolutions historiques des régimes politiques. Les États fédéraux sont exclus du champ d'application de la présente étude mais il est néanmoins intéressant de rappeler l'évolution en Allemagne. Le pouvoir central a surtout insisté, au lendemain du traumatisme de la seconde guerre mondiale, sur l'interdiction de toute forme de discrimination. Aussi l'article 3 alinéa 3 de la Loi fondamentale dispose-t-il que « Nul ne doit être discriminé ni privilégié en raison de (...) sa langue ». L'Allemagne a ratifié la Charte des langues régionales et minoritaires en 1998 avec cette précision suivante : «Les langues minoritaires au sens de la Charte européenne des langues régionales ou minoritaires en République fédérale d'Allemagne sont le danois, le haut sorabe, le bas sorabe, le frison septentrional et le frison saterois, ainsi que la langue rom des Sintis et Roms de nationalité allemande ; la langue régionale au sens de la Charte en République fédérale d'Allemagne est le bas allemand ${ }^{4}$. En droit espagnol et italien, il faut distinguer deux niveaux de protection. Les langues régionales font l'objet d'une reconnaissance par les constitutions nationales, interprétées par ailleurs par les juridictions

\footnotetext{
${ }^{4}$ Déclarations consignées dans une lettre de la Représentation Permanente de l'Allemagne, en date du 16 septembre 1998, remise au Secrétaire Général lors du dépôt de l'instrument de ratification, le 16 septembre 1998, https:/www.coe.int/fr/web/conventions/search-on-treaties/-/conventions/treaty/148/declarations
} 
constitutionnelles (A) mais leur aménagement est le fait des statuts d'autonomie, qui ont une valeur supérieure à celle des lois (B).

\section{A. Une reconnaissance par les constitutions nationales}

La Constitution espagnole consacre clairement le multilinguisme dans le cadre de ses communautés autonomes (Alcaraz 2019). En vertu de l'article 3,

1. Le castillan est la langue espagnole officielle de l'État. Tous les Espagnols ont le devoir de le connaitre et le droit de l'utiliser.

2. Les autres langues espagnoles sont aussi officielles dans leurs communautés autonomes respectives conformément à leurs statuts.

3. La richesse de la diversité linguistique de l'Espagne est un patrimoine culturel qui fait l'objet d'un respect et d'une protection spéciales.

La notion de « langue officielle » a été précisée par le Tribunal constitutionnel dans un arrêt du 26 juin $1986^{5}$. Saisi d'un recours en inconstitutionnalité intenté par le gouvernement central contre certaines dispositions de la loi basque de normalisation de l'usage de l'euskera, le Tribunal a jugé que «bien que la Constitution ne définisse pas, mais présume ce qu'est une langue officielle, la réglementation établie en la matière permet d'affirmer qu'est officielle une langue, indépendamment de sa réalité et de son poids comme phénomène social, quand elle est reconnue par les pouvoirs publics comme moyen normal de communication en leur sein et entre eux, et dans leurs relations avec les personnes privées, avec pleine valeur et effets juridiques ».

La comparaison avec la Constitution italienne est pertinente, puisque celle-ci associe également unité et indivisibilité de la République et reconnaissance des langues au pluriel (Torretta 2014 ; Passaglia 2019 ). Selon son article 3 également,

1) Tous les citoyens ont une même dignité sociale et sont égaux devant la loi sans distinction de sexe, de race, de langue, de religion, d'opinions politiques, de conditions personnelles et sociales.

L'article 6 énonce quant à lui que « La République protège par des mesures particulières les minorités linguistiques ». C'est la Corte costituzionale qui a précisé ce que recouvrait concrètement ce concept de protection. À partir d'une décision de $1982^{6}$, elle a jugé qu'il appartenait au législateur de chercher une «protection minimale ». Standard de protection minimale qui permet notamment aux membres d'une minorité d'employer leur langue maternelle dans leurs relations avec les administrations publiques et de recevoir des réponses dans la même langue. En l'espèce, il s'agissait de la minorité slovène : "Cette protection "minimale", même dans les relations avec les tribunaux locaux, permet déjà aux membres de la minorité slovène d'utiliser leur langue maternelle et de recevoir des réponses des autorités dans leur langue maternelle : dans les communications verbales, soit directement, soit par l'intermédiaire d'un interprète; dans la correspondance, avec le texte italien accompagné d'une traduction en slovène ». À cette protection générale s'ajoute des protections spécifiques dérogatoires énoncées dans certains statuts régionaux (voir infra). Dans ces deux pays, il faut lire les articles des constitutions de manière combinée avec les statuts d'autonomie des régions en Italie et communautés autonomes en Espagne.

\footnotetext{
${ }^{5}$ STC n ${ }^{\circ}$ SSTC 82/1986. Voir Milian-Massana 1992.

${ }^{6}$ Corte costituzionale, sentenza $n^{\circ} 28,20$ janvier 1982.
} 


\section{B. Un aménagement par les statuts d'autonomie}

Il faut au préalable préciser que dans les États régionaux qui accordent la co-officialité au niveau local, il existe des degrés divers dans cet aménagement. Les régions italiennes et les communautés autonomes espagnoles ne jouissent pas toutes de cette reconnaissance. Le catalan est co-officiel dans les Communautés autonomes de Catalogne et des iles Baléares, le valencien dans la Communauté autonome de Valence, le galicien dans la Communauté autonome de Galice et l'euskera dans les Communautés autonomes du pays basque et de Navarre. D'autres langues sont en revanche dépourvues de statut, comme l'asturien, l'aragonais, l'andalou ou le léonais. L'Italie est dans une situation similaire, un statut de co-officialité étant organisé dans certaines régions à statut spécial, dans le Trentin-Haut-Adige (avec l'allemand), le Val d'Aoste (avec le français) et le Frioul-Vénétie-Julienne (avec le slovène).

Le Statut d'autonomie des Baléares tel que modifié en 2007 comporte un article intitulé « la langue distinctive $\gg$. Il dispose :

1) La langue catalane, particulière aux iles Baléares, bénéficie à côté du castillan, du statut de langue officielle.

2) Tous ont le droit de la connaitre et de l'utiliser, et nul ne pourra être victime de discrimination en raison de la langue.

3) Les institutions des iles Baléares garantissent l'usage normal et officiel des deux langues, prennent les mesures nécessaires pour assurer leur connaissance et créent les conditions permettant d'arriver à l'égalité entière des deux langues quant aux droits des citoyens des iles Baléares.

Avant sa censure partielle par le Tribunal constitutionnel, l'article 6 du statut de Catalogne adopté le 30 septembre 2005 disposait que :

1) La langue propre de la Catalogne est le catalan. En tant que tel, le catalan est la langue utilisée habituellement et de préférence par les administrations publiques et les médias publics de Catalogne. En outre, le catalan est normalement utilisé comme langue véhiculaire et d'apprentissage dans l'enseignement.

2) Le catalan est la langue officielle de la Catalogne, de même que le castillan, qui est la langue officielle de l'État espagnol. Toutes les personnes ont le droit d'utiliser les deux langues officielles, et les citoyens de Catalogne ont le droit et le devoir de les connaitre. Les pouvoirs publics de Catalogne doivent mettre en place les mesures nécessaires pour faciliter l'exercice de ces droits et le respect de ce devoir. Conformément aux dispositions de l'article 32, il ne peut y avoir de discrimination en raison de l'utilisation de l'une ou l'autre langue.

3) La Generalitat et l'État doivent mener les actions nécessaires pour la reconnaissance $d u$ caractère officiel du catalan dans l'Union européenne et la présence et l'utilisation du catalan dans les organismes internationaux et dans les traités internationaux à contenu culturel ou linguistique.

4) La Generalitat doit promouvoir la communication et la coopération avec les autres communautés et territoires possédant un patrimoine linguistique commun avec la Catalogne. À ces effets, la Generalitat et l'État, selon le cas, peuvent adhérer à des conventions, des traités et autres mécanismes de collaboration pour la promotion et la diffusion du catalan à l'étranger.

5) La langue occitane, qui porte le nom d'aranais en Aran, est la langue propre de ce territoire et elle est une langue officielle en Catalogne, conformément aux dispositions du présent Statut et des lois de normalisation linguistique. 
Le premier paragraphe a été déclaré contraire à la Constitution par les juges constitutionnels dans un arrêt du 28 juin $2010^{7}$. Si le catalan demeure la langue d'usage "normale» en Catalogne, elle ne saurait être qualifiée de « préférentielle » dans l'administration et les médias.

En Italie, la Corte Costituzionale, dans la décision précitée de 1982, tout en affirmant que l'Italien est la langue officielle, précise que des exceptions sont prévues dans certains statuts, « textuellement par l'article 84 du Statut de la région du Trentin-Haut-Adige (aujourd'hui 99 de la loi de consolidation approuvée par le décret présidentiel $n^{\circ} 670$ du 31 août 1972) et par l'article 38 du Statut spécial du Val d'Aoste ». Le premier dispose en effet que «Dans la Région, la langue allemande est à parité avec l'italien qui est la langue officielle de l'État. La langue italienne prévaut dans les actes ayant caractère législatif et dans les cas où le présent Statut prévoit une rédaction bilingue $»$.

Le statut spécial pour le Val d'Aoste énonce, dans son article 38 :

1) La langue française et la langue italienne sont à parité en Vallée d'Aoste.

2) Les actes publics peuvent être rédigés dans l'une ou l'autre langue, à l'exception des actes de l'autorité judiciaire, qui sont rédigés en italien.

3) Les administrations de l'État prennent à leur service dans la Vallée, autant que possible, des fonctionnaires originaires de la Région ou qui connaissent le français.

Au Royaume-Uni, la reconnaissance du gaélique ne survint que tardivement. En vertu du Small Landholders Scotland Act de 1911, un membre au moins du Tribunal foncier d'Écosse devait parler la langue gaélique. Le statut juridique de la langue gaélique écossaise est celui d'une « langue protégée », à l'instar du gallois au pays de Galles ${ }^{8}$. De manière générale, les lois dites de dévolution britanniques auront un effet positif sur la promotion du gallois. La reconnaissance de celui-ci est entrée, comme souvent, par les portes de la justice (Welsh Courts Act de 1942) et de l'éducation (Education Act de 1944). Toute une série de lois vont avoir pour objet de permettre l'utilisation de la langue galloise dans plusieurs secteurs publics jusqu'à sa reconnaissance officielle par le Welsh Language Act de 1993 ${ }^{9}$. Mais ladite loi s'applique à 1'Administration locale du pays de Galles, non aux services gouvernementaux britanniques. Si Westminster a officialisé le gallois, il n'a pas pour autant offert un quelconque droit linguistique à la population du pays de Galles. La Loi sur le gouvernement du pays de Galles de 1998 (Government of Wales Act), prévoit que les langues anglaise et galloise soient traitées sur un pied d'égalité, mais « dans la limite du raisonnable », expression largement soumise aux vents de multiples interprétations.

Le cas français, unique en son genre du point de vue de l'indifférence à l'égard des langues régionales, a poussé Véronique Bertile à utiliser l'expression de «clandestinité constitutionnelle des langues régionales » (op. cit : 90).

\section{Imposer l'unilinguisme de l'État}

En vertu de l'article 2, alinéa 2 de la Constitution de la $\mathrm{V}^{\mathrm{e}}$ République française, « la langue de la République est le français ». Il faut d'emblée souligner que cet article n'a pas été inscrit en 1958 mais en 1992, à l'occasion d'une révision constitutionnelle portant essentiellement sur la ratification du traité de Maastricht. L'objectif du pouvoir constituant était de faire barrage

\footnotetext{
${ }^{7}$ Tribunal constitucional, Sentencia 31/2010, de 28 de junio de 2010, BOE núm. 172, de 16 de julio de 2010, pp. $1-491$.

${ }^{8}$ Voir, pour une approche sociolinguistique, Cardinal 1990.

${ }^{9}$ La première loi sur la langue galloise, également dénommée Welsh Language Act, avait été adoptée en 1967. Elle avait pour objet d'accorder, dans les tribunaux du pays de Galles, l'usage égal de l'anglais et du gallois.
} 
aux langues étrangères, notamment à l'anglais. Mais dans les faits, il aura surtout pour conséquence de minimiser les langues régionales. Depuis l'ordonnance de Villers-Cotterêt du 10 août 1539 confirmant le français comme langue officielle, l'arsenal normatif n'a cessé de confirmer le dogme de l'unilinguisme, sanctuarisé dans la Constitution et confirmé par la loi du 4 août 1994, dite « loi Toubon », précisant que le français est la langue de l'enseignement, du travail, des échanges et des services publics. Cette posture en lien avec le jacobinisme centralisateur, unique en droit comparé (A) laisse peu de perspectives optimistes pour les défenseurs des langues régionales en France (B).

\section{A. Le culte constitutionnel de l'unicité de la langue}

Formellement, on relèvera tout d'abord que les expressions, courantes en droit comparé, de « langue officielle » ou « langue nationale » ne sont pas utilisées dans la Constitution française. C'est le lien entre la langue et le régime politique qui a été privilégié, ce qui donne d'autant plus de force à cette exclusivité linguistique. De nombreux commentateurs ont souligné le caractère quasi iconique de cette disposition, qui devient, avec l'hymne, la devise et le drapeau, l'un des symboles forts de la Nation française. Symbole d'autant plus fort quand on le met en perspective avec certains discours révolutionnaires, qui entendaient faire du français la langue de la révolution, la langue de la liberté. Le discours le plus célèbre est sans aucun doute celui de l'Abbé Grégoire, prononcé à une époque où chaque région française utilisait un patois, le français de l'Ile-de-France étant réservé à une certaine élite. C'est donc dans un souci égalitariste que l'abbé plaida pour la diffusion de «l'idiome de la liberté », afin que les cœurs soient « à l'unisson » :

Nous n'avons plus de provinces et nous avons encore 30 Patois qui en rappellent les noms. [...] On peut assurer sans exagération qu'au moins 6 millions de Français, surtout dans les campagnes, ignorent la langue nationale ; qu'un nombre égal est à peu près incapable de soutenir une conversation suivie ; qu'en dernier résultat, le nombre de ceux qui la parlent purement n'excède pas 3 millions, et probablement le nombre de ceux qui l'écrivent correctement est encore moindre. Ainsi, avec 30 patois différents, nous sommes encore pour le langage à la Tour de Babel, tandis que pour la liberté, nous formons l'avant-garde des nations. Notre langue et nos cœurs doivent être à l'unisson. [...] Tout ce qu'on vient de dire appelle la conclusion que, pour extirper tous les préjugés, développer toutes les vérités, tous les talents, toutes les vertus, fondre tous les citoyens dans la masse nationale, simplifier le mécanisme et faciliter le jeu de la machine politique, il faut identité de langage. [...] La Nation, entièrement rajeunie par vos soins, triomphera de tous les obstacles et rien ne ralentira le cours d'une révolution qui doit améliorer le sort de l'espèce humaine. ${ }^{10}$

Le discours du religieux ne poursuivait pas le but de la destruction des patois mais la volonté de supprimer les inégalités en instaurant une langue commune qui permettrait d'effacer les castes, dans la droite ligne de l'œuvre révolutionnaire. La fin des privilèges, la fin des castes pour lesquelles les révolutionnaires avaient sacrifié leur vie devait passer par une langue commune, universelle, qui ne se diviserait plus en la langue des élus sociaux d'une part et les patois des miséreux d'autre part. Mais les temps ont changé. Cette même problématique peut conduire, aujourd'hui, à produire les mêmes effets pervers que ceux qui étaient alors combattus. Il est évident qu'il s'agit d'un discours à replacer dans un contexte et à ne pas utiliser comme arme contre la reconnaissance, de nos jours, des langues régionales. Mais il est vrai que cette

10 Discours disponible sur le site de l'Assemblée nationale : http://www2.assemblee-nationale.fr/decouvrir-lassemblee/histoire/grands-discours-parlementaires/l-abbe-gregoire-4-juin-1794 
philosophie de l'unité de la langue pour œuvrer à l'unité de la République est toujours très prégnante en France, comme en atteste la jurisprudence du Conseil constitutionnel, notamment à partir de la décision statut de la Corse du 9 mai $1991^{11}$. Demeurée célèbre pour avoir refusé l'expression " peuple corse, composante du peuple français », la décision fut le résultat d'une saisine qui estimait que l'article 53 de la loi du 13 mai 1991 était contraire à la Constitution. Celui-ci disposait que « l'assemblée adopte (...) un plan de développement de l'enseignement de la langue et de la culture corses, prévoyant notamment les modalités de l'insertion de cet enseignement dans le temps scolaire ». Au nom de la rupture d'égalité entre les élèves du territoire français, le Conseil constitutionnel répond au législateur que « cet enseignement n'est pas contraire au principe d'égalité dès lors qu'il ne revêt pas un caractère obligatoire $»$. Quelques années plus tard, à l'article 7 de la loi du 22 janvier 2002 qui énonçait plus prudemment que « la langue corse est une matière enseignée dans le cadre de l'horaire normal des écoles maternelles et élémentaires de Corse », la haute juridiction répondit que cet enseignement « ne saurait revêtir pour autant un caractère obligatoire ni pour les élèves, ni pour les enseignants $»^{12}$.

C'est également à propos du créole que le gardien de la Constitution a eu l'occasion de rappeler que l'indivisibilité de la République entrainait - exigeait - l'unicité de la langue. Dans sa décision du 9 avril 1996 sur la Loi organique portant statut d'autonomie de la Polynésie française, le Conseil constitutionnel a jugé que le français était la langue qui s'imposait «aux personnes morales de droit public et aux personnes de droit privé dans l'exercice d'une mission de service public, ainsi qu'aux usagers dans leurs relations avec les administrations et services publics $\gg{ }^{13}$. Il reprendra à peu près la même formulation dans la décision du 15 juin 1999 relative à la Charte européenne des langues régionales ou minoritaires ${ }^{14}$. Affirmant clairement de nouveau le principe de «l'unicité du peuple français », le Conseil a fermement établi le principe d'interdiction, celui des éventuels droits linguistiques accordés à une quelconque communauté : "Considérant que ces principes fondamentaux s'opposent à ce que soient reconnus des droits collectifs à quelque groupe que ce soit, défini par une communauté d'origine, de culture, de langue ou de croyance ». Si l'indivisibilité de la République s'oppose bien à l'existence juridique d'une quelconque «minorité » ou «communauté », elle n'est pas nécessairement signe de l'imposition d'une unité superficielle et autoritaire. Le droit comparé apporte la preuve irréfutable que ce principe, également présenté comme sacro-saint dans certaines constitutions européennes, n'est pas contradictoire avec la reconnaissance d'autres langues. De manière générale, cette adéquation jacobine « une République : une langue » découle de la philosophie de construction de la République française, plus fondée sur la recherche de l'égalité que sur le mythe de la liberté. À l'époque révolutionnaire, cette quête avait un sens et il ne saurait être question de la dévaloriser. Mais les temps ont évolué et en Europe, l'expression des langues régionales apparait comme une richesse et non une menace. Or l'esprit français, politique et juridique comme en témoignent les réticences actuelles à l'égard du principe de différenciation, demeure marqué par la tradition jacobine qui nie et combat la diversité territoriale.

\section{B. Les perspectives : l'exemple de la constitutionnalisation de la Corse comme préalable à la défense d'une langue régionale}

La jurisprudence «statut de la Corse » du Conseil constitutionnel a été en large partie frappée d'obsolescence par la réforme constitutionnelle de 2003. Les principes d'unicité du peuple français et de la langue française sont mis à mal depuis ladite révision. La France en tant

\footnotetext{
${ }^{11}$ Décision n ${ }^{\circ}$ 91-290 DC du 9 mai 1991, Loi portant statut de la collectivité territoriale de Corse.

${ }^{12}$ Décision $n^{\circ} 2001-454$ DC du 17 janvier 2002 Loi relative à la Corse.

${ }^{13}$ Décision n ${ }^{\circ}$ 96-373 DC du 9 avril 1996 Loi organique portant statut d'autonomie de la Polynésie française.

${ }^{14}$ Décision n ${ }^{\circ}$ 99-412 DC du 15 juin 1999 Charte européenne des langues régionales ou minoritaires.
} 
que République ne saurait s'appréhender de deux manières : avec ou sans l'Outre-mer. Une France qui demeurerait unitaire par le prisme d'une lecture limitée à la métropole ; une France régionale quand la lecture s'élargit à l'Outre-Mer, et quasi-fédérale quand elle englobe la Nouvelle-Calédonie. Les larges dérogations accordées aux territoires ultramarins ne justifient plus que l'on continue de mettre l'ile métropolitaine à l'écart de dérogations au moins analogues.

L'entrée remarquée des langues régionales dans l'article 75-1 de la Constitution en 2008 n'a eu que des effets symboliques. Certes non négligeables, comme tous les symboles, mais dénués d'effectivité. La haute instance a d'ailleurs souligné son caractère purement déclaratoire: «Considérant (...) que cet article n'institue pas un droit ou une liberté que la Constitution garantit ; que sa méconnaissance ne peut donc être évoquée à l'appui d'une question prioritaire de constitutionnalité sur le fondement de l'article 61-1 de la Constitution ${ }^{15}$.

À l'époque de l'entrée, dans l'article 75-1, de l'élévation des langues régionales au rang de « patrimoine de la France » en 2008, certains espoirs avaient pu être légitimement nourris. Cette précision n'est pas, loin sans faut, le sésame pour la ratification de la Charte européenne des langues régionales ou minoritaires ${ }^{16}$, qui, en l'état actuel de la jurisprudence du Conseil constitutionnel $^{17}$, comporte des clauses jugées contraires à la Constitution. Tout l'enjeu se situe dans cette problématique: une reconnaissance plus effective de la langue corse, jusqu'à l'hypothèse, ultime, de la co-officialité, pourrait peut-être passer par la ratification de la charte. Laquelle ne pourra intervenir qu'après une révision de la Constitution.

En 1999, faisant la synthèse de ses décisions antérieures ${ }^{18}$, le Conseil constitutionnel a donc estimé que la Charte européenne des langues régionales ou minoritaires ne pouvait, au vu des principes d'unicité du peuple français, d'indivisibilité de la République et d'égalité des citoyens, être ratifiée : «la Charte européenne des langues régionales ou minoritaires, en ce qu'elle confère des droits spécifiques à des "groupes" de locuteurs de langues régionales ou minoritaires, à l'intérieur de "territoires" dans lesquels ces langues sont pratiquées, porte atteinte aux principes constitutionnels d'indivisibilité de la République, d'égalité devant la loi et d'unicité du peuple français ${ }^{19}$. Le Conseil se défendant au passage de mépriser les langues régionales: parmi les engagements souscrits par la France, précise-t-il, «la plupart, au demeurant, se bornent à reconnaitre des pratiques déjà mises en œuvre par la France en faveur des langues régionales ».

Parmi les arguments qui plaident en faveur d'une ratification, figure celui de l'évolution du contexte. Honorer de la ratification un texte déjà signé ne se pose, aujourd'hui, plus dans les mêmes termes qu'en 1999. La réforme constitutionnelle de 2003 - dont certains aspects ont été affinés en 2008 -, rappelons-le, a considérablement modifié la vision jacobine de notre République qui reconnait à présent l'existence de «populations» en son sein, les particularismes locaux et les nécessités des adaptations normatives. L'actuel projet de loi

\footnotetext{
${ }^{15}$ Décision 2011-130 QPC du 20 mai 2011, Mme Cécile L. et autres précitée.

${ }^{16}$ Voir la table ronde (Jean-Eric Gicquel, Ferdinand Mélin-Soucramanien, Michel Verpeaux et Jean-Marie Woehrling) organisée par la commission des lois le 29 novembre 2011 sur les implications constitutionnelles de la question de la ratification de la charte européenne des langues régionales ou minoritaires, http://www.languesregionales.org/Commission-des-lois-Table-ronde.

${ }^{17}$ Décision 99-412 DC du 15 juin 1999 précitée.

${ }^{18}$ Décisions 94-345 DC du 29 juillet 1994 sur la loi relative à l'emploi de la langue française et 96-373 DC du 9 avril 1996 sur la loi organique portant statut d'autonomie de la Polynésie française.

${ }^{19}$ Il s'agissait là, au demeurant, d'une affirmation très discutable car la lettre de la Charte n'imposait nullement, même si elle l'encourageait, que des droits spécifiques fussent reconnus à des groupes (voir Guy Carcassonne 1998).
} 
constitutionnelle $^{20}$ et le projet de loi «3D» en préparation ${ }^{21}$ vont dans le même sens, en permettant la différenciation des compétences mais aussi des normes. Plaider pour un statut de la langue corse, tant que le statut de la Corse n'est pas revisité serait une erreur, et la censure du Conseil constitutionnel quasi certaine.

Il faut, pour commencer, rapprocher la mention des langues régionales de l'article 2. L'évocation des langues, l'une à l'article 2, l'autre à l'article 75-1, symbolise un grand écart géographique loin d'être inconsciemment désiré. Lors de la révision du 21 juillet 2008, les députés eux-mêmes avaient envisagé d'introduire un second alinéa à l'article 2, du type « les langues régionales appartiennent à son patrimoine ». Mais cette retouche ne serait que cosmétique, car dénuée de portée normative (y compris de possibilité d'implications normatives). Le droit comparé nous enseigne que davantage de reconnaissance des langues régionales (encouragée, il est vrai, par le caractère composé des États cités plus haut) ne signifie pas affaiblissement de la langue dominante.

La meilleure réforme est, de toute évidence, la plus logique : la Corse ne pourra obtenir une meilleure reconnaissance de sa langue que lorsqu'elle aura elle-même un vrai statut. La méthode de la modification est donc impérieuse : pas de statut particulier de la langue corse sans un statut clairement dérogatoire préalablement établi pour la Corse. La mention des langues régionales dès l'article 2 , même sous une forme purement déclaratoire (du style « elle encourage la promotion des langues régionales ", avec renvoi à une loi organique pour la définition et la liste desdites langues), combinée à une mention dans un article ${ }^{22}$, voire un titre exclusivement réservé à la Corse, serait bien plus qu'une pierre posée à l'édifice de la reconnaissance de la langue corse : elle en serait en quelque sorte la prémisse «idéale ${ }^{23}$, du fait de la supériorité de la Constitution sur les autres normes. Mais pas indispensable : de nombreuses avancées pourraient néanmoins être opérées, pour la langue corse comme pour toutes les autres langues régionales, à travers l'œuvre du législateur.

Cette étude comparative permet de mettre en évidence la singularité de la France au sein d'une Europe qui s'enorgueillit de se définir à travers la richesse de sa diversité. Il est un fait, objectif, absolument incontestable. La République française, bien qu'officiellement «décentralisée » en vertu de sa constitution, est foncièrement, quasi ontologiquement centralisée. L'histoire de notre République peut aussi, peut-être avant tout, se raconter par le prisme jacobin qui nie et combat la diversité territoriale. Si aucun de ses chefs d'État n'a jamais renversé la tendance décentralisatrice initiée par le prestigieux premier d'entre eux, personne n'a pour autant opéré une révolution girondine et l'actuel, malgré la promesse de campagne de son « pacte girondin », ne sera sans doute pas de ceux-là.

\footnotetext{
${ }^{20}$ L'article 15 du projet de loi constitutionnelle présenté au Conseil des ministres du 28 août 2019 introduit pour les collectivités territoriales une double possibilité de différenciation : différenciation des compétences, d'une part, en permettant que la loi puisse "prévoir que certaines collectivités territoriales exercent des compétences, en nombre limité, dont ne disposent pas l'ensemble des collectivités de la même catégorie»; différenciation des normes applicables, d'autre part, en permettant que les collectivités territoriales ou leurs groupements puissent "déroger, pour un objet limité, aux dispositions législatives ou réglementaires qui régissent l'exercice de leurs compétences, éventuellement après une expérimentation autorisée dans les mêmes conditions ».

$213 \mathrm{D}$ pour décentralisation, différenciation et déconcentration. Voir https://www.cohesionterritoires.gouv.fr/projet-de-loi-3d-decentralisation-differenciation-et-deconcentration

${ }^{22}$ Le projet de loi constitutionnelle précité, dont la discussion au Parlement a été maintes fois repoussée, prévoit d'insérer un nouvel article 72-5 disposant que « Les lois et règlements peuvent comporter des règles adaptées aux spécificités liées à son insularité ainsi qu'à ses caractéristiques géographiques, économiques ou sociales ».

${ }^{23}$ Nous nous permettons de renvoyer à notre rapport sur le statut constitutionnel de la Corse, remis au président de l'Assemblée de Corse et disponible sur le site internet de cette dernière : https://www.isula.corsica/assemblea/Pour-un-statut-constitutionnel-de-la-Corse-consultez-le-rapportMastor a2.html.
} 


\section{Bibliographie}

Alcaraz H., 2019, "Quel statut juridique pour quel type de reconnaissance? Le cas de l'Espagne ", in Gorgorza A. et Mastor W., Les langues régionales et la construction de l'État en Europe, Lextenso, collection Grands colloques, pp. 83-102.

Bertile V., 2008, Langues régionales ou minoritaires et Constitution. France, Espagne et Italie, Bruylant, Coll. Droit public comparé et européen, $516 \mathrm{p}$.

Carcassonne G., 1998, Rapport au Premier ministre sur la compatibilité entre la Charte européenne des langues régionales ou minoritaires et la Constitution, septembre 1998, http://www.ladocumentationfrancaise.fr/rapports-publics/984001697/.

Cardinal L., 1990. «Politiques linguistiques et mobilisations ethnolinguistiques au Canada et en Grande-Bretagne depuis les années $1990 »$, Cultures et conflits, $\mathrm{n}^{\circ} 79-80$, automne/hiver 2010, p. 37-54.

Gorgorza A. et Mastor W., 2019, Les langues régionales et la construction de l'État en Europe, Lextenso, collection Grands colloques, 318 p.

Milian-Massana, A., 1992, «Droits linguistiques et droits fondamentaux en Espagne », Revue générale de droit, $\mathrm{n}^{\circ} 23$ (4), pp. 561-581, https://www.erudit.org/fr/revues/rgd/1992v23-n4-rgd04378/1057025ar.pdf.

Torretta A., 2014, «Diritti fondamentali e protezione delle "istanze collettive di diversità" : il caso delle minoranze linguistiche », Diritto pubblico comparato ed europeo, p. 695 et suiv.

Passaglia P., 2019, « La protection des langues minoritaires et régionales en droit italien », in Gorgorza A. et Mastor W., Les langues régionales et la construction de l'État en Europe, Lextenso, collection Grands colloques, pp. 129-150. 


\section{GLOTTOPOL}

Revue de sociolinguistique en ligne

Comité de rédaction : Michaël Abecassis, Salih Akin, Sophie Babault, Claude Caitucoli, Véronique Castellotti, Régine Delamotte, Robert Fournier, Stéphanie Galligani, Emmanuelle Huver, Normand Labrie, Foued Laroussi, Benoit Leblanc, Fabienne Leconte, Gudrun Ledegen, Danièle Moore, Clara Mortamet, Alioune Ndao, Isabelle Pierozak, Gisèle Prignitz.

Rédactrice en chef : Clara Mortamet.

Comité scientifique : Claudine Bavoux, Michel Beniamino, Jacqueline Billiez, Philippe Blanchet, Pierre Bouchard, Ahmed Boukous, Pierre Dumont, Jean-Michel Eloy, Françoise Gadet, Monica Heller, Caroline Juilliard, Jean-Marie Klinkenberg, Jean Le Dû $(\dagger)$, Marinette Matthey, Jacques Maurais, Marie-Louise Moreau, Robert Nicolaï, Didier de Robillard, Paul Siblot, Claude Truchot, Daniel Véronique.

\section{Comité de lecture pour ce numéro :}

Salih Akin, Carmen Alén Garabato, Sophie Babault, Philippe Blanchet, Henri Boyer, Véronique Castellotti, Marisa Cavalli, Jean-François De Pietro, Didier de Robillard, Alain Di Meglio, Ksenija Djordjevic, Jean Michel Eloy, Pascale Erahrt, Véronique Fillol, Monica Heller, Robert Fournier, Normand Labrie, Hervé Lieutard, Jean Le Dû (†), Marinette Matthey.

http://glottopol.univ-rouen.fr

ISSN : 1769-7425 